\title{
Chaotic continua of (continuum-wise) expansive homeomorphisms and chaos in the sense of $\mathrm{Li}$ and Yorke
}

\author{
by
}

\author{
Hisao K a t o (Hiroshima)
}

\begin{abstract}
A homeomorphism $f: X \rightarrow X$ of a compactum $X$ is expansive (resp continuum-wise expansive) if there is $c>0$ such that if $x, y \in X$ and $x \neq y$ (resp. if $A$ is a nondegenerate subcontinuum of $X)$, then there is $n \in \mathbb{Z}$ such that $d\left(f^{n}(x), f^{n}(y)\right)$ $>c$ (resp. $\left.\operatorname{diam} f^{n}(A)>c\right)$. We prove the following theorem: If $f$ is a continuum-wise expansive homeomorphism of a compactum $X$ and the covering dimension of $X$ is positive $(\operatorname{dim} X>0)$, then there exists a $\sigma$-chaotic continuum $Z=Z(\sigma)$ of $f(\sigma=\mathrm{s}$ or $\sigma=\mathrm{u})$, i.e. $Z$ is a nondegenerate subcontinuum of $X$ satisfying: (i) for each $x \in Z, V^{\sigma}(x ; Z)$ is dense in $Z$, and (ii) there exists $\tau>0$ such that for each $x \in Z$ and each neighborhood $U$ of $x$ in $X$, there is $y \in U \cap Z$ such that $\liminf _{n \rightarrow \infty} d\left(f^{n}(x), f^{n}(y)\right) \geq \tau$ if $\sigma=\mathrm{s}$, and $\liminf _{n \rightarrow \infty} d\left(f^{-n}(x), f^{-n}(y)\right) \geq \tau$ if $\sigma=\mathrm{u}$; in particular, $W^{\sigma}(x) \neq W^{\sigma}(y)$. Here

$V^{\mathrm{s}}(x ; Z)=\{z \in Z \mid$ there is a subcontinuum $A$ of $Z$ such that

$$
\left.x, z \in A \text { and } \lim _{n \rightarrow \infty} \operatorname{diam} f^{n}(A)=0\right\},
$$

$V^{\mathrm{u}}(x ; Z)=\{z \in Z \mid$ there is a subcontinuum $A$ of $Z$ such that

$$
\begin{aligned}
& W^{\mathrm{s}}(x)=\left\{x^{\prime} \in X \mid \lim _{n \rightarrow \infty} d\left(f^{n}(x), f^{n}\left(x^{\prime}\right)\right)=0\right\}, \quad \text { and } \\
& W^{\mathrm{u}}(x)=\left\{x^{\prime} \in X \mid \lim _{n \rightarrow \infty} d\left(f^{-n}(x), f^{-n}\left(x^{\prime}\right)\right)=0\right\} .
\end{aligned}
$$$$
\left.x, z \in A \text { and } \lim _{n \rightarrow \infty} \operatorname{diam} f^{-n}(A)=0\right\},
$$

As a corollary, if $f$ is a continuum-wise expansive homeomorphism of a compactum $X$ with $\operatorname{dim} X>0$ and $Z$ is a $\sigma$-chaotic continuum of $f$, then for almost all Cantor sets $C \subset Z, f$ or $f^{-1}$ is chaotic on $C$ in the sense of $\mathrm{Li}$ and Yorke according as $\sigma=\mathrm{s}$ or u). Also, we prove that if $f$ is a continuum-wise expansive homeomorphism of a compactum $X$ with $\operatorname{dim} X>0$ and there is a finite family $\mathbb{F}$ of graphs such that $X$ is $\mathbb{F}$-like, then each chaotic continuum of $f$ is indecomposable. Note that every expansive homeomorphism is continuum-wise expansive.

1991 Mathematics Subject Classification: Primary 54H20, 54F50; Secondary 54E40, $54 \mathrm{~B} 20$.

Key words and phrases: expansive homeomorphism, continuum-wise expansive homeomorphism, stable and unstable sets, scrambled set, chaotic in the sense of Li and Yorke, independent, indecomposable continuum. 
1. Introduction. All spaces under consideration are assumed to be metric. By a compactum, we mean a compact metric space. A connected nondegenerate compactum is called a continuum. Let $X$ be a compactum. Then $X$ has dimension $\leq n$, denoted by $\operatorname{dim} X \leq n$, if for every $\gamma>0$ there is a covering $\mathcal{U}$ of $X$ by open sets with diameter $\leq \gamma$ such that ord $\mathcal{U} \leq n+1$, i.e., every point of $X$ belongs to at most $n+1$ sets of $\mathcal{U}$. If $\operatorname{dim} X \leq n$ and $\operatorname{dim} X \leq n-1$ is not true, then $\operatorname{dim} X=n$. It is known that a compactum $X$ is 0 -dimensional (i.e., $\operatorname{dim} X=0$ ) if and only if each component of $X$ is a single point. We refer the reader to [8] for the properties of dimension of separable metric spaces.

Let $\mathbb{Z}$ be the set of all integers. Let $X$ be a compactum with metric $d$. A homeomorphism $f: X \rightarrow X$ is expansive ([27] or [6]) if there is $c>0$ such that if $x, y \in X$ and $x \neq y$, then there is an integer $n=n(x, y) \in \mathbb{Z}$ such that

$$
d\left(f^{n}(x), f^{n}(y)\right)>c .
$$

Expansiveness does not depend on the choice of the metric $d$ of $X$. This property has frequent applications in topological dynamics, ergodic theory and continuum theory (see [1], [2], [5]-[7], [9]-[15], [18], [19], [23], [25]-[29]) for the properties of expansive homeomorphisms). For example, in [23] Mañé proved that if $f$ is an expansive homeomorphism of a compactum $X$, then $\operatorname{dim} X<\infty$ and every minimal set of $f$ is 0-dimensional. In [25] Plykin showed that there exist plane continua admitting expansive homeomorphisms. Such continua are called "lakes of Wada"; their topological properties were studied in continuum theory. In [7], Hiraide proved that if $f$ is an expansive homeomorphism of a compact closed surface $M$, then $f$ is a pseudo-Anosov map, and that the 2-sphere admits no expansive homeomorphism.

A homeomorphism $f$ of a compactum $X$ is continuum-wise expansive [16] if there is $c>0$ such that if $A$ is a nondegenerate subcontinuum of $X$, then there is an integer $n=n(A) \in \mathbb{Z}$ such that

$$
\operatorname{diam} f^{n}(A)>c,
$$

where $\operatorname{diam} B=\sup \{d(x, y) \mid x, y \in B\}$ for any $B \subseteq X$. Similarly, $f$ is positively continuum-wise expansive if there is $c>0$ such that if $A$ is a nondegenerate subcontinuum of $X$, then there is $n \geq 0$ such that $\operatorname{diam} f^{n}(A)>c$. Such a $c>0$ is called an expansive constant for $f$. By the definitions, we can easily see that every expansive homeomorphism is continuum-wise expansive. However, the converse is not true (see [16]-[18] for the properties of continuum-wise expansive homeomorphisms).

By many examples, we know that expansive homeomorphisms and continuum-wise expansive homeomorphisms are "chaotic", and compacta admitting such homeomorphisms may have considerably complicated dy- 
namical structure. The following notion of "chaos" due to Li and Yorke [22] is well known. Let $f: X \rightarrow X$ be a map of a compactum $X$. A subset $S$ of $X$ is called a scrambled set of $f$ if for any $x, y \in S$ with $x \neq y$,

(1) $\limsup _{n \rightarrow \infty} d\left(f^{n}(x), f^{n}(y)\right)>0$,

(2) $\liminf _{n \rightarrow \infty} d\left(f^{n}(x), f^{n}(y)\right)=0$, and

(3) $\lim \sup _{n \rightarrow \infty} d\left(f^{n}(x), f^{n}(p)\right)>0$ for any periodic point $p$ of $f$.

If there is a scrambled set $S$ of $f$ that is uncountable, then we say that $f$ is chaotic (on $S$ ) in the sense of Li and Yorke [22]. Also, the following notion of chaos is well known (see [4]). A map $f: X \rightarrow X$ of a compactum $X$ with metric $d$ has sensitive dependence on initial conditions on a closed subset $A$ of $X$ if there is $\tau>0$ such that if $x \in A$ and $U$ is any neighborhood of $x$ in $X$, then there is $y \in U \cap A$ and $n \geq 0$ such that $d\left(f^{n}(x), f^{n}(y)\right)>\tau$. If $A=X$, we simply say that $f$ has sensitive dependence on initial conditions.

In this paper, we define the notion of a "chaotic continuum" of a homeomorphism $f$, which plays an important role in determining the behavior of the dynamical system of $f$, and we prove that if $f$ is a continuum-wise expansive homeomorphism of a compactum $X$ with $\operatorname{dim} X>0$, then there exists a chaotic continuum $Z$ of $f$; as a consequence, we show that either $f$ or $f^{-1}$ is then chaotic in the sense of Li and Yorke on almost all Cantor sets $C \subset Z$. Actually, we show that continuum-wise expansive homeomorphisms yield slightly stronger chaos than the chaos in the sense of $\mathrm{Li}$ and Yorke. Also, we prove that if $f$ is a continuum-wise expansive homeomorphism of a compactum $X$ and there is a finite family $\mathbb{F}$ of graphs such that $X$ is $\mathbb{F}$-like, then each chaotic continuum of $f$ is indecomposable.

2. Chaotic continua of homeomorphisms. Let $X$ be a metric space with metric $d$. The hyperspaces of $X$ are the sets

$$
\begin{aligned}
2^{X} & =\{A \mid A \text { is a nonempty compact subset of } X\} \text { and } \\
C(X) & =\left\{A \in 2^{X} \mid A \text { is connected }\right\}
\end{aligned}
$$

with the Hausdorff metric $d_{\mathrm{H}}$, i.e., $d_{\mathrm{H}}(A, B)=\inf \left\{\varepsilon>0 \mid U_{\varepsilon}(A) \supset B\right.$ and $\left.A \subset U_{\varepsilon}(B)\right\}$, where $U_{\varepsilon}(A)$ denotes the $\varepsilon$-neighborhood of $A$ in $X$. Note that if $X$ is a compactum, then $2^{X}$ and $C(X)$ are compact metric spaces, and moreover if $X$ is a continuum, then $2^{X}$ and $C(X)$ are path connected (e.g., see [24]). Also, for $A, B \subset X$, put $d(A, B)=\inf \{d(a, b) \mid a \in A$ and $b \in B\}$.

Let $f: X \rightarrow X$ be a homeomorphism of a compactum $X$. For $x \in X$, the stable set $W^{\mathrm{s}}(x)$ and the unstable set $W^{\mathrm{u}}(x)$ of $f$ are defined as follows:

$$
\begin{aligned}
& W^{\mathrm{s}}(x)=\left\{y \in X \mid \lim _{n \rightarrow \infty} d\left(f^{n}(x), f^{n}(y)\right)=0\right\} \quad \text { and } \\
& W^{\mathrm{u}}(x)=\left\{y \in X \mid \lim _{n \rightarrow \infty} d\left(f^{-n}(x), f^{-n}(y)\right)=0\right\} .
\end{aligned}
$$


For each $x \in X$ and each closed subset $Z$ of $X$, put

$$
W^{\mathrm{s}}(x ; Z)=Z \cap W^{\mathrm{s}}(x) \text { and } W^{\mathrm{u}}(x ; Z)=Z \cap W^{\mathrm{u}}(x) .
$$

Note that we do not assume $x \in Z$. Also, the continuum-wise stable and unstable sets $V^{\mathrm{s}}(x)$ and $V^{\mathrm{u}}(x)$ of $f$ are defined as follows:

$V^{\mathrm{s}}(x)=\{y \in X \mid$ there is $A \in C(X)$ such that

$$
\left.x, y \in A \text { and } \lim _{n \rightarrow \infty} \operatorname{diam} f^{n}(A)=0\right\} \quad \text { and }
$$

$V^{\mathrm{u}}(x)=\{y \in X \mid$ there is $A \in C(X)$ such that

$$
\left.x, y \in A \text { and } \lim _{n \rightarrow \infty} \operatorname{diam} f^{-n}(A)=0\right\} .
$$

Similarly, for each closed subset $Z$ of $X$ and $x \in Z$, put

$V^{\mathrm{s}}(x ; Z)=\{y \in Z \mid$ there is $A \in C(Z)$ such that

$$
\left.x, y \in A \text { and } \lim _{n \rightarrow \infty} \operatorname{diam} f^{n}(A)=0\right\} \quad \text { and }
$$

$V^{\mathrm{u}}(x ; Z)=\{y \in Z \mid$ there is $A \in C(Z)$ such that

$$
\left.x, y \in A \text { and } \lim _{n \rightarrow \infty} \operatorname{diam} f^{-n}(A)=0\right\} .
$$

Clearly, $V^{\sigma}(x ; X)=V^{\sigma}(x)$, and if $x \in Z$, then $W^{\sigma}(x, Z) \supset V^{\sigma}(x ; Z)$, for both $\sigma=\mathrm{s}$ and $\sigma=\mathrm{u}$. Note that $V^{\sigma}(x ; Z)$ is connected, and $W^{\sigma}(x ; Z)$ and $V^{\sigma}(x ; Z)$ are not always closed in $X$.

For each $0<\delta<\varepsilon$, consider the following subsets of $C(X)$ :

$\mathbb{V}^{\mathrm{s}}(\delta ; \varepsilon)=\left\{A \in C(X) \mid \operatorname{diam} A=\delta\right.$ and $\operatorname{diam} f^{n}(A) \leq \varepsilon$ for each $\left.n \geq 0\right\}$,

$\mathbb{V}^{\mathrm{u}}(\delta ; \varepsilon)=\left\{A \in C(X) \mid \operatorname{diam} A=\delta\right.$ and $\operatorname{diam} f^{-n}(A) \leq \varepsilon$ for each $\left.n \geq 0\right\}$,

$\mathbb{V}^{\mathrm{s}}=\left\{A \in C(X) \mid \lim _{n \rightarrow \infty} \operatorname{diam} f^{n}(A)=0\right\} \quad$ and

$\mathbb{V}^{\mathrm{u}}=\left\{A \in C(X) \mid \lim _{n \rightarrow \infty} \operatorname{diam} f^{-n}(A)=0\right\}$.

Let $f$ be a homeomorphism of a compactum $X$ with $\operatorname{dim} X>0$. Then a subcontinuum $Z$ of $X$ is a $\sigma$-chaotic continuum of $f$ (where $\sigma=\mathrm{s}$ or u) if

(i) for each $x \in Z, V^{\sigma}(x ; Z)$ is dense in $Z$, and

(ii) there is $\tau>0$ such that for each $x \in Z$ and each neighborhood $U$ of $x$ in $X$, there is $y \in U \cap Z$ such that $\liminf _{n \rightarrow \infty} d\left(f^{n}(x), f^{n}(y)\right) \geq \tau$ in case $\sigma=\mathrm{s}$, and $\liminf \operatorname{in}_{n \rightarrow \infty} d\left(f^{-n}(x), f^{-n}(y)\right) \geq \tau$ in case $\sigma=\mathrm{u}$.

Note that (ii) implies that $W^{\sigma}(x) \neq W^{\sigma}(y)$, in particular, $V^{\sigma}(x) \neq$ $V^{\sigma}(y)$. Similarly, $Z$ is a weakly $\sigma$-chaotic continuum of $f$ if

(i) for each $x \in Z, V^{\sigma}(x ; Z)$ is dense in $Z$, and

(ii) $^{\prime}$ for each $x \in Z$ and each neighborhood $U$ of $x$ in $X$, there is $y \in U \cap Z$ such that $V^{\sigma}(x ; Z) \neq V^{\sigma}(y ; Z)$.

Clearly, chaotic subcontinua are weakly chaotic. Note that (i) implies that $W^{\sigma}(x ; Z)$ is dense in $Z$. In the definition of a weakly chaotic continuum, 
(ii)' can be replaced with

(ii)" there are $x, y \in Z$ such that $V^{\sigma}(x ; Z) \neq V^{\sigma}(y ; Z)$

because (i) and (ii)" together imply (i) and (ii)'.

If $Z$ is a (weakly) $\sigma$-chaotic continuum of $f$ for $\sigma=\mathrm{s}$ or $\mathrm{u}$, we simply say that $Z$ is a (weakly) chaotic continuum of $f$.

By the very definition, (weakly) chaotic continua of $f$, if they exist, play a very important role in the dynamics of $f$. Here we are interested in the following question: Does every expansive (or continuum-wise expansive) homeomorphism admit a chaotic continuum? In Section 3, we give an affirmative answer to this question. Also, it turns out that for such homeomorphisms, weakly chaotic continua are chaotic continua.

From continuum theory, we know that the inverse limit construction yields complicated spaces and maps from simple ones. Let $f: X \rightarrow X$ be a map of a compactum $X$. Consider the inverse limit space

$$
(X, f)=\left\{\left(x_{n}\right)_{n=0}^{\infty} \mid x_{n} \in X \text { and } f\left(x_{n+1}\right)=x_{n} \text { for each } n\right\} .
$$

Then $(X, f)$ is a compact metric space with metric

$$
\widetilde{d}(\widetilde{x}, \widetilde{y})=\sum_{n=0}^{\infty} d\left(x_{n}, y_{n}\right) / 2^{n}, \quad \text { where } \widetilde{x}=\left(x_{n}\right)_{n=0}^{\infty}, \widetilde{y}=\left(y_{n}\right)_{n=0}^{\infty} \in(X, f) .
$$

Define $\tilde{f}:(X, f) \rightarrow(X, f)$ by

$$
\widetilde{f}\left(\left(x_{0}, x_{1}, x_{2}, \ldots\right)\right)=\left(f\left(x_{0}\right), f\left(x_{1}\right), f\left(x_{2}\right), \ldots\right) \quad\left(=\left(f\left(x_{0}\right), x_{0}, x_{1}, \ldots\right)\right),
$$

for $\left(x_{0}, x_{1}, x_{2}, \ldots\right) \in(X, f)$. Then $\tilde{f}$ is a homeomorphism, called the shift map of $f$. Note that for an onto map $f: G \rightarrow G$ of a graph $G$ (= compact connected 1-dimensional polyhedron $G$ ), $f$ has sensitive dependence on initial conditions if and only if $\widetilde{f}:(G, f) \rightarrow(G, f)$ is (positively) continuumwise expansive (see $[16,(3.9)])$.

(2.1) Example. (1) Let $S^{1}$ be the unit circle and let $f: S^{1} \rightarrow S^{1}$ be the natural covering map with degree 2 . Then $X=\left(S^{1}, f\right)$ is the 2-adic solenoid and the shift map $\tilde{f}:\left(S^{1}, f\right) \rightarrow\left(S^{1}, f\right)$ is expansive (see [29]) and positively continuum-wise expansive. By $[18,(3.9)]$, for each $\widetilde{x} \in X, V^{\mathrm{s}}(\widetilde{x})=$ $\{\widetilde{x}\}, \operatorname{dim} W^{\mathrm{s}}(\widetilde{x})=0$ and $W^{\mathrm{u}}(\widetilde{x})=V^{\mathrm{u}}(\widetilde{x})$ is the path component of $X$ containing $\widetilde{x}$. In the 2 -adic solenoid $X$, each path component is a composant of $X$, and hence $V^{\mathrm{u}}(\widetilde{x})$ is a composant of the indecomposable continuum $X$ (see Section 5 for the definitions of an indecomposable continuum and a composant). Since each composant of a continuum is dense (see [20, p. 209]), we see that $X$ is itself a weakly u-chaotic continuum, and hence a chaotic continuum, of $f$. On the other hand, $f$ has no (weakly) s-chaotic continuum, because $\operatorname{dim} W^{\mathrm{s}}(\widetilde{x})=0$ and $V^{\mathrm{s}}(\widetilde{x})=\{\widetilde{x}\}$ for each $\widetilde{x} \in X$. 
(2) In general, (weakly) chaotic continua are not equal to the total space. Consider the one-point union $G=\left(S^{1}, *\right) \vee(I, 1)$ where $I=[0,1]$. Define $g: G \rightarrow G$ by $g \mid S^{1}=f, g(0)=0$ and $g(*)=*$ and $g(I)=G$. We can choose $g: G \rightarrow G$ so that $\widetilde{g}:(G, g) \rightarrow(G, g)$ is expansive [11, Theorem 4.3]. Then the chaotic continuum $Z=\left(S^{1}, g \mid S^{1}\right)$ of $\widetilde{g}$ is a proper subset of $(G, g)$.

(3) Let $I=[0,1]$. For each $n=2,3, \ldots$, let $f_{n}: I \rightarrow I$ be defined by

$$
f_{n}(t)= \begin{cases}n t-s & \text { if } s \text { is even, } \\ -n t+s+1 & \text { if } s \text { is odd }\end{cases}
$$

for $s=0,1, \ldots, n-1$ and $t \in[s / n,(s+1) / n]$. Then $K(n)=\left(I, f_{n}\right)$ is the Knaster chainable continuum of order $n$. Then the shift map $\widetilde{f}_{n}:\left(I, f_{n}\right) \rightarrow$ $\left(I, f_{n}\right)$ is a (positively) continuum-wise expansive homeomorphism, but it is not expansive. Moreover, $X=\left(I, f_{n}\right)$ is a u-chaotic continuum of $\widetilde{f}_{n}$, because $V^{\mathrm{u}}(\widetilde{x})$ is the composant of $X$ containing $\widetilde{x}$ (see (3.15)). On the other hand, $\widetilde{f}_{n}$ has no (weakly) s-chaotic continuum, because $V^{\mathrm{s}}(\widetilde{x})=\{\widetilde{x}\}$ for each $\widetilde{x} \in X$.

(4) Let $f: T^{2} \rightarrow T^{2}$ be an Anosov diffeomorphism, say $\left(\begin{array}{ll}2 & 1 \\ 1 & 1\end{array}\right)$, on the 2-dimensional torus $T^{2}$. Note that $f$ is expansive ([26]). Then $T^{2}$ itself is a (weakly) $\sigma$-chaotic continuum of $f$ for both $\sigma=\mathrm{s}$ and $\sigma=\mathrm{u}$ (e.g., see [4] and (3.15) below).

(5) Let $G_{i}=S_{i}^{1} \vee I_{i}(i=1,2)$ be two copies of $G$ as in (2). Consider the one-point union $H=\left(G_{1}, 0_{1}\right) \vee\left(G_{2}, 0_{2}\right)$, where $0_{i} \in I_{i}$. Let $\overrightarrow{\mathbf{a}}$ and $\overrightarrow{\mathbf{b}}$ be orientations of the circles $S_{1}^{1}$ and $S_{2}^{1}$, respectively. Also, let $\overrightarrow{\mathbf{c}}=\overrightarrow{0_{1} 1_{1}}$ and $\overrightarrow{\mathbf{d}}=\overrightarrow{0_{2} 1_{2}}$ be the orientations of the intervals $I_{1}$ and $I_{2}$, respectively. Define $h: H \rightarrow H$ by

$$
\begin{aligned}
& \overrightarrow{\mathbf{a}} \rightarrow \overrightarrow{\mathbf{a}}+\overrightarrow{\mathbf{a}} \\
& \overrightarrow{\mathbf{b}} \rightarrow \overrightarrow{\mathbf{b}}+\overrightarrow{\mathbf{b}} \\
& \overrightarrow{\mathbf{c}} \rightarrow \overrightarrow{\mathbf{c}}+\overrightarrow{\mathbf{a}}-\overrightarrow{\mathbf{c}}+\overrightarrow{\mathbf{d}}+\overrightarrow{\mathbf{b}}-\overrightarrow{\mathbf{d}}+\overrightarrow{\mathbf{c}} \\
& \overrightarrow{\mathbf{d}} \rightarrow \overrightarrow{\mathbf{d}}+\overrightarrow{\mathbf{b}}-\overrightarrow{\mathbf{d}}+\overrightarrow{\mathbf{c}}+\overrightarrow{\mathbf{a}}-\overrightarrow{\mathbf{c}}+\overrightarrow{\mathbf{d}}
\end{aligned}
$$

Then the shift map $\widetilde{h}:(H, h) \rightarrow(H, h)$ is expansive and positively continuum-wise expansive. Let $W=(H, h)-\left(\left(G_{1}, h \mid G_{1}\right) \cup\left(G_{2}, h \mid G_{2}\right)\right)$. Then $W$ is open and dense in $(H, h)$. Since for each $\widetilde{x} \in(H, h), W^{\mathrm{u}}(\widetilde{x})=V^{\mathrm{u}}(\widetilde{x})$ is the path component of $(H, h)$ containing $\widetilde{x}$, we see that if $\widetilde{x} \in W$, then $V^{\mathrm{u}}(\widetilde{x})=W^{\mathrm{u}}(\widetilde{x})$ is dense in $X=(H, h)$ (compare this condition with the condition (i) of the definition of a weakly chaotic continuum), and for each $\widetilde{x} \in(H, h)$ and each neighborhood $U$ of $\widetilde{x}$ in $X$ there is $\widetilde{y} \in U$ such that $W^{\mathrm{u}}(\widetilde{x}) \neq W^{\mathrm{u}}(\widetilde{y})$ (see the condition (ii)' of the definition of a weakly chaotic continuum). However, $(H, h)$ is not a weakly chaotic continuum of $\widetilde{h}$, because if $\widetilde{x} \in\left(G_{i}, h \mid G_{i}\right)$, then $V^{\mathrm{u}}(\widetilde{x})$ is dense in $\left(G_{i}, h \mid G_{i}\right)$, but not in $(H, h)$, because 
$\left(G_{i}, h \mid G_{i}\right) \varsubsetneqq(H, h)$. Note that $X$ is indecomposable, and if $\widetilde{x} \in\left(G_{i}, h \mid G_{i}\right)$, then $V^{\mathrm{u}}(\widetilde{x})=W^{\mathrm{u}}(\widetilde{x})$ is not equal to the composant of $X$ containing $\widetilde{x}$.

3. Existence of chaotic continua of continuum-wise expansive homeomorphisms. In this section, we prove the main theorem (3.6) of this paper which implies the existence of a chaotic continuum of a continuumwise expansive homeomorphism. We need the following lemmas.

(3.1) Lemma ([16, (2.1)]). Let $f$ be a continuum-wise expansive homeomorphism of a compactum $X$ with an expansive constant $c>0$. Let $c \geq \varepsilon$ $>0$. If $A \in C(X)$ and $\operatorname{diam} f^{n}(A) \leq \varepsilon$ for each $n \geq 0$ (resp. $\operatorname{diam} f^{-n}(A) \leq$ $\varepsilon$ for each $n \geq 0$ ). then $A \in \mathbb{V}^{\mathrm{s}}$, i.e., $\lim _{n \rightarrow \infty} \operatorname{diam} f^{n}(A)=0$ (resp. $A \in \mathbb{V}^{\mathrm{u}}$, i.e., $\left.\lim _{n \rightarrow \infty} \operatorname{diam} f^{-n}(A)=0\right)$.

(3.2) Lemma (see the proof of $[16,(2.3)]$ ). Let $f$ be a continuum-wise expansive homeomorphism of a compactum $X$ with an expansive constant $c>0$ and let $0<\varepsilon \leq c / 2$. Then there is $\delta>0$ such that if $A$ is any nondegenerate subcontinuum of $X$ such that $\operatorname{diam} A \leq \delta$ and $\operatorname{diam} f^{m}(A)$ $\geq \varepsilon$ for some integer $m \in \mathbb{Z}$, then one of the following conditions holds:

(a) If $m \geq 0$, then $\operatorname{diam} f^{n}(A) \geq \delta$ for each $n \geq m$. More precisely, for each $x \in f^{n}(A)$ there is a subcontinuum $B$ of $A$ such that $x \in f^{n}(B)$, $\operatorname{diam} f^{j}(B) \leq \varepsilon$ for $0 \leq j \leq n$ and $\operatorname{diam} f^{n}(B)=\delta$.

(b) If $m<0$, then $\operatorname{diam} f^{-n}(A) \geq \delta$ or each $n \geq-m$. More precisely, for each $x \in f^{-n}(A)$ there is a subcontinuum $B$ of $A$ such that $x \in f^{-n}(B)$, $\operatorname{diam} f^{-j}(B) \leq \varepsilon$ for $0 \leq j \leq n$ and $\operatorname{diam} f^{-n}(B)=\delta$.

(3.3) Lemma $([16,(2.5)])$. If $f$ is a continuum-wise expansive homeomorphism of a compactum $X$ with $\operatorname{dim} X>0$, then there is a nondegenerate subcontinuum $A$ of $X$ such that either $A \in \mathbb{V}^{\mathrm{s}}$ or $A \in \mathbb{V}^{\mathrm{u}}$.

(3.4) Lemma $([16,(2.4)])$. Let $f, c, \varepsilon, \delta$ be as in (3.2). Then for each $\gamma>0$ there is $N=N(\gamma) \geq 0$ such that if $A \in C(X)$ and $\operatorname{diam} A \geq \gamma$, then either $\operatorname{diam} f^{n}(A) \geq \delta$ for each $n \geq N$ or $\operatorname{diam} f^{-n}(A) \geq \delta$ for each $n \geq N$.

By a simple modification of the proof of Brouwer's Reduction Theorem [8, p. 161], we obtain the following.

(3.5) Proposition (a modification of Brouwer's Reduction Theorem). In a space with countable basis, let $\left\{K_{\lambda}\right\}$ be a family of closed sets with this property: if $K_{1}, K_{2}, K_{3}, \ldots$ is a sequence of members of $\left\{K_{\lambda}\right\}$ such that

$$
K_{1} \supset K_{2} \supset K_{3} \supset \ldots,
$$

then there is a member $K_{\infty}$ of $\left\{K_{\lambda}\right\}$ such that $\bigcap_{n=1}^{\infty} K_{n} \supset K_{\infty}$. Then there exists an irreducible set $(=$ a minimal set $)$ in $\left\{K_{\lambda}\right\}$.

Now, we prove the main theorem of this paper. 
(3.6) THEOREM. If $f$ is a continuum-wise expansive homeomorphism of a compactum $X$ with $\operatorname{dim} X>0$, then there exists a chaotic continuum of $f$.

Proof. Let $c>0$ be an expansive constant for $f$ and let $0<\varepsilon \leq c / 2$. Choose $\delta>0$ as in (3.2). By (3.2) and (3.3), $\mathbb{V}^{\sigma}(\delta ; \varepsilon) \neq \emptyset$ for $\sigma=\mathrm{s}$ or u. We may assume that $\sigma=\mathrm{s}$.

For each pair $(y ; Y)$ with $y \in Y \in C(X)$, consider the following condition:

$\mathbf{P}(y ; Y) \quad \operatorname{Cl}\left(V^{\mathrm{s}}(y ; Y)\right)=Y$, and for each $n \geq 0$, there is $A_{n} \in \mathbb{V}^{\mathrm{s}}(\delta ; \varepsilon)$ such that $f^{n}(y) \in A_{n}$ and $f^{-n}\left(A_{n}\right) \subset V^{\mathrm{s}}(y ; Y)$, i.e., $y \in f^{-n}\left(A_{n}\right)$ $\subset Y$.

We now show that if a pair $(y ; Y)$ satisfies $\mathbf{P}(y ; Y)$ and $y^{\prime} \in Y$, then for each $n \geq 0$ there is $A_{n}^{\prime} \in \mathbb{V}(\delta ; \varepsilon)$ such that $f^{n}\left(y^{\prime}\right) \in A_{n}^{\prime}$ and $f^{-n}\left(A_{n}^{\prime}\right) \subset$ $V^{\mathrm{s}}\left(y^{\prime} ; Y\right)$. Choose a sequence $\left\{y_{i}\right\}_{i=1}^{\infty}$ in $V^{\mathrm{s}}(y ; Y)$ such that $\lim _{i \rightarrow \infty} y_{i}=y^{\prime}$, and, for each $i \geq 1$, choose $B_{i} \in \mathbb{V}^{\mathrm{s}}\left(y, y_{i} ; Y\right)=\left\{A \in C(X) \mid y, y_{i} \in A\right.$ and $\left.\lim _{i \rightarrow \infty} \operatorname{diam} f^{i}(A)=0\right\}$. Fix $n$. For each $i \geq 1$, choose a subcontinuum $D_{i}$ of $X$ such that diam $D_{i}=\delta, f^{n}\left(y_{i}\right) \in D_{i}$ and $D_{i} \subset A_{n} \cup f^{n}\left(B_{i}\right)$, where $A_{n}$ is as in the condition $\mathbf{P}(y ; Y)$. Since $D_{i} \in \mathbb{V}^{\mathrm{s}}$, by (3.2) we see that $D_{i} \in V^{\mathrm{s}}(\delta ; \varepsilon)$. We may assume that $\lim _{i \rightarrow \infty} D_{i}=A_{n}^{\prime}$. Then $A_{n}^{\prime} \in \mathbb{V}^{\mathrm{s}}(\delta ; \varepsilon)$, $f^{n}\left(y^{\prime}\right) \in A_{n}^{\prime}$ and $f^{-n}\left(A_{n}^{\prime}\right) \subset Y$. Note that, in general, we cannot conclude that $V^{\mathrm{s}}\left(y^{\prime} ; Y\right)$ is dense in $Y=\mathrm{Cl}\left(V^{\mathrm{s}}(y ; Y)\right)$.

Now, define

$$
\Gamma=\{Y \in C(X) \mid \text { there is } y \in Y \text { such that } \mathbf{P}(y ; Y) \text { holds }\} .
$$

First, we prove that $\Gamma \neq \emptyset$. Since $\mathbb{V}^{\mathrm{s}}(\delta ; \varepsilon) \neq \emptyset$, we choose $x \in A \in$ $\mathbb{V}^{\mathrm{s}}(\delta ; \varepsilon)$. Let $\alpha(x, f)$ be the $\alpha$-limit set of $x$, i.e., $\alpha(x, f)$ is the set of all subsequential limits of $\left\{f^{-n}(x)\right\}_{n=0}^{\infty}$. Note that $f(\alpha(x, f))=\alpha(x, f)$ is a closed subset of $X$. Choose $y \in \alpha(x, f)$ and put $Y=\mathrm{Cl}\left(V^{\mathrm{s}}(y ; X)\right)$. Since $\mathbb{V}^{\mathrm{s}}(\delta ; \varepsilon)$ is closed in $C(X)$ and hence compact, by $(3.2)$ the pair $(y ; Y)$ satisfies $\mathbf{P}(y ; Y)$. This implies that $\Gamma \neq \emptyset$.

Next, we prove that if $Y_{1} \supset Y_{2} \supset \ldots$ is a sequence in $\Gamma$, then there is $Y_{\infty} \in \Gamma$ such that $\bigcap_{i=1}^{\infty} Y_{i} \supset Y_{\infty}$. For each $n \geq 1$, choose $y_{i} \in Y_{i}$ such that $\left(y_{i} ; Y_{i}\right)$ satisfies $\mathbf{P}\left(y_{i} ; Y_{i}\right)$. We may assume that $\lim _{i \rightarrow \infty} y_{i}=y^{\prime}$. Fix $n$. Choose $B_{i, n} \in \mathbb{V}^{\mathrm{s}}(\delta ; \varepsilon)$ such that $f^{n}\left(y_{i}\right) \in B_{i, n}$ and $f^{-n}\left(B_{i, n}\right) \subset V^{\mathrm{s}}\left(y_{i} ; Y_{i}\right) \subset$ $Y_{i}$. We may assume that $\lim _{i \rightarrow \infty} B_{i, n}=B_{n} \in \mathbb{V}^{\mathrm{s}}(\delta ; \varepsilon)$. Note that $f^{-n}\left(B_{n}\right) \subset$ $V^{\mathrm{s}}\left(y^{\prime} ; \bigcap_{i=1}^{\infty} Y_{i}\right)$. Put $Y_{\infty}=\operatorname{Cl}\left(V^{\mathrm{s}}\left(y^{\prime} ; \bigcap_{i=1}^{\infty} Y_{i}\right)\right)$. Then $\left(y^{\prime} ; Y_{\infty}\right)$ satisfies $\mathbf{P}\left(y^{\prime} ; Y_{\infty}\right)$, which implies that $\bigcap_{i=1}^{\infty} Y_{i} \supset Y_{\infty} \in \Gamma$.

By (3.5), we can choose a minimal element $Z$ of $\Gamma$. If $z \in Z$, then by minimality, $(z ; Z)$ satisfies $\mathbf{P}(z ; Z)$, for if not, we can choose an element $Z^{\prime}=\mathrm{Cl}\left(V^{\mathrm{s}}(z ; Z)\right)$ of $\Gamma$ such that $Z^{\prime} \varsubsetneqq Z$.

Now, we prove the following claim. 
Claim (*): Suppose that $f: X \rightarrow X$ is a continuum-wise expansive homeomorphism of a compactum $X$ with $\operatorname{dim} X>0$ and $Z$ is a subcontinuum of $X$ satisfying the following conditions:

(a) if $x \in Z$, then $V^{\mathrm{s}}(x ; Z)$ is dense in $Z$, and

(b) if $x \in Z$ and $n \geq 0$ is any natural number, then there exists $A_{n} \in$ $\mathbb{V}^{\mathrm{s}}(\delta ; \varepsilon)$ such that $f^{n}(x) \in A_{n}$ and $f^{-n}\left(A_{n}\right) \subset Z$.

Then $Z$ is an s-chaotic continuum of $f$.

First, we show that there is $\varrho>0$ such that if $z \in Z$, then there is $z^{\prime} \in Z$ such that $d\left(f^{n}(z), f^{n}\left(z^{\prime}\right)\right)>\varrho$ for each $n \geq 0$. By (3.4), we can choose $N \geq 0$ such that if $A \in \mathbb{V}^{\mathrm{s}}(\delta / 4 ; \varepsilon)$, then $\operatorname{diam} f^{-N}(A) \geq \delta$. Without los of generality, we may assume that $N=1$ (if necessary, consider $f^{\prime}=f^{N}$ instead of $f$ ). By (b), for each $n \geq 0$ there is $A_{n} \in V^{\mathrm{s}}(\delta ; \varepsilon)$ such that $f^{n}(z) \in A_{n}$ and $f^{-n}\left(A_{n}\right) \subset Z$. Fix $n$. Since $\operatorname{diam} A_{n}=\delta$, we can choose a subcontinuum $B_{n}$ of $A_{n}$ such that $d\left(f^{n}(z), B_{n}\right) \geq \delta / 4$ and $\operatorname{diam} B_{n} \geq \delta / 4$. Since $\operatorname{diam} f^{-1}\left(B_{n}\right) \geq \delta$, we can choose a subcontinuum $B_{n-1}$ of $f^{-1}\left(B_{n}\right)$ such that $d\left(f^{n-1}(z), B_{n-1}\right) \geq \delta / 4$ and $\operatorname{diam} B_{n-1} \geq \delta / 4$. If we continue this procedure, we obtain a sequence $B_{n}, B_{n-1}, \ldots, B_{1}, B_{0}$ of subcontinua of $X$ such that

$$
B_{j-1} \subset f^{-1}\left(B_{j}\right), \quad d\left(f^{j}(z), B_{j}\right) \geq \delta / 4 \quad \text { and } \quad \operatorname{diam} B_{j} \geq \delta / 4
$$

for $j=n, n-1, \ldots, 1,0$.

Choose $z_{n} \in B_{0}$. Thus we get a sequence $\left\{z_{n}\right\}$ of points of $V^{\mathrm{s}}(z ; Z)$. Since $Z$ is compact, there is a subsequence such that $\lim _{i \rightarrow \infty} z_{n_{i}}=z^{\prime} \in Z$. Since $d\left(f^{i}(z), f^{i}\left(z_{n}\right)\right) \geq \delta / 4$ for each $0 \leq i \leq n$, we see that $d\left(f^{n}(z), f^{n}\left(z^{\prime}\right)\right) \geq$ $\delta / 4=\varrho>0$ for each $n \geq 0$.

Let $x \in Z$ and $U$ be any neighborhood of $x$ in $X$. Let $\tau>0$ with $\tau<\varrho=\delta / 4$. By the above argument, there exists $x^{\prime}$ in $Z$ such that $d\left(f^{n}(x), f^{n}\left(x^{\prime}\right)\right) \geq \delta / 4>\tau>0$ for each $n \geq 0$. Since $V^{\mathrm{s}}\left(x^{\prime} ; Z\right)$ is dense in $Z$, there is $x^{\prime \prime} \in U \cap V^{\mathrm{s}}\left(x^{\prime} ; Z\right)$. Since $\lim _{n \rightarrow \infty} d\left(f^{n}\left(x^{\prime}\right), f^{n}\left(x^{\prime \prime}\right)\right)=0$, we see that $\liminf \operatorname{in}_{n \rightarrow \infty} d\left(f^{n}(x), f^{n}\left(x^{\prime \prime}\right)\right)>\tau$. Hence $Z=\mathrm{Cl}\left(V^{\mathrm{s}}(z ; Z)\right)$ is an s-chaotic continuum of $f$. The proof of the corresponding statement for $\sigma=\mathrm{u}$ is similar.

(3.7) Proposition. Let $f$ be a continuum-wise expansive homeomorphism of a compactum $X$ with $\operatorname{dim} X>0$, let $c, \varepsilon$ be positive numbers as in (3.2), and let $Z$ be a subcontinuum of $X$. Then the following are equivalent.

(1) $Z$ is a $\sigma$-chaotic continuum of $f$.

(2) $Z$ is a weakly $\sigma$-chaotic continuum of $f$.

(3) $Z$ satisfies the following conditions (a) and (b) (see Claim (*) in the proof of (3.6)). 
(a) if $x \in Z$, then $V^{\sigma}(x ; Z)$ is dense in $Z$, and

(b) there is $\varepsilon>\tau>0$ such that if $\sigma=\mathrm{s}$ (resp. $\sigma=\mathrm{u}), x \in Z$ and $n \geq 0$ is any natural number, then there is $A_{n} \in V^{\mathrm{s}}(\tau ; \varepsilon)$ (resp. $A_{-n} \in V^{\mathrm{u}}(\tau ; \varepsilon)$ ) such that $f^{n}(x) \in A_{n}$ and $f^{-n}\left(A_{n}\right) \subset Z$ (resp. $f^{-n}(x) \in A_{-n}$ and $\left.f^{n}\left(A_{-n}\right) \subset Z\right)$.

Proof. By the proof of (3.6), (3) implies (1). Clearly, (1) implies (2). We must show that (2) implies (3). Suppose that $\sigma=$ s. Let $\delta$ be as in (3.2). Since there are $x, y \in Z$ such that $V^{\mathrm{s}}(x ; Z) \neq V^{\mathrm{s}}(y ; Z), Z$ is not an element of $\mathbb{V}^{\mathrm{s}}$. By (3.1), $\lim \sup _{n \rightarrow \infty} \operatorname{diam} f^{i}(Z)>\varepsilon$. If there is $n \geq 0$ such that $\operatorname{diam} f^{n}(Z) \leq \delta$, we can choose $m>n$ such that $\operatorname{diam} f^{m}(Z)>\varepsilon$. By (3.2), $\operatorname{diam} f^{i}(Z) \geq \delta$ for each $i \geq m$. Consequently, we can choose $\tau>0$ such that $\operatorname{diam} f^{i}(Z)>\tau$ for each $i \geq 0$. Let $x \in Z$ and let $n$ be any natural number. Since $V^{\mathrm{s}}(x ; Z)$ is dense in $Z$, we can choose a subcontinuum $B_{n}$ of $Z$ such that $x \in B_{n} \subset V^{\mathrm{s}}(x ; Z)$ and $\operatorname{diam} f^{n}\left(B_{n}\right)>\tau$. Choose a subcontinuum $A_{n}$ of $f^{n}\left(B_{n}\right)$ such that $f^{n}(x) \in A_{n}$ and $\operatorname{diam} A_{n}=\tau$. Then $A_{n}(n \geq 0)$ satisfies the desired conditions.

(3.8) Remark. (1) We see that if $Z$ is an s-chaotic continuum of a homeomorphism $f$, then $E=\left\{(x, y) \in Z \times Z \mid \liminf _{n \rightarrow \infty} d\left(f^{n}(x), f^{n}(y)\right)\right.$ $\leq \tau\}$ is not an $F_{\sigma}$-set in $Z \times Z$.

(2) In (3.6), we can conclude that if $\mathbb{V}^{\sigma}$ contains a nondegenerate subcontinuum, then $f$ has a $\sigma$-chaotic continuum.

Also, by the proof of (3.6), we obtain the following.

(3.9) Proposition. Let $f$ be a continuum-wise expansive homeomorphism of a compactum $X$ and let $\varepsilon$ be as in (3.2). Suppose that for some $x \in X$ and $\sigma=\mathrm{s}($ resp. $\sigma=\mathrm{u})$, there is $\tau>0$ and, for each $n \geq 0$, there is $A_{n} \in \mathbb{V}^{\mathrm{s}}(\tau ; \varepsilon)$ with $f^{n}(x) \in A_{n}\left(\right.$ resp. $A_{-n} \in \mathbb{V}^{\mathrm{u}}(\tau ; \varepsilon)$ with $\left.f^{-n}(x) \in A_{-n}\right)$. Then $f$ has a $\sigma$-chaotic continuum contained in $\mathrm{Cl}\left(V^{\sigma}(x)\right)$. In particular, if $x \in X$ is a periodic point of $f$, then there exists a $\sigma$-chaotic continuum of $f$, contained in $\mathrm{Cl}\left(V^{\sigma}(x)\right)$ (for $\sigma=\mathrm{s}$ or $\left.\mathrm{u}\right)$.

Pr o o f. We may assume that $x$ is a fixed point of $f$, i.e., $f(x)=x$. Choose a nondegenerate subcontinuum $A$ of $X$ such that $x \in A$ and diam $A=\gamma<\delta$, where $c, \varepsilon$ and $\delta$ are as in (3.2). By (3.4), we can choose $N \geq 0$ such that either (1) $\operatorname{diam} f^{n}(A) \geq \delta$ for $n \geq N$ or (2) $\operatorname{diam} f^{-n}(A) \geq \delta$ for $n \geq N$.

We assume that (1) holds. Choose a subcontinuum $A_{1}$ of $f^{N}(A)$ such that $x \in A_{1}$ and $\operatorname{diam} A_{1}=\gamma$. By (3.4), $\operatorname{diam} f^{N}\left(A_{1}\right) \geq \delta$. We can choose a nondegenerate subcontinuum $A_{2}$ of $f^{N}\left(A_{1}\right)$ such that $x \in A_{2}$ and $\operatorname{diam} A_{2}=$ $\gamma$. If we continue this procedure, we obtain a sequence $\left\{A_{n}\right\}$ of subcontinua of $X$. By (3.2), $\max \left\{\operatorname{diam} f^{-i}\left(A_{n}\right) \mid 0 \leq i \leq n N\right\} \leq \varepsilon$. We may assume that $\lim _{n \rightarrow \infty} A_{n}=B$. Then $x \in B \in V^{\mathrm{u}}(\gamma ; \varepsilon)$. By $(3.1), B \in \mathbb{V}^{\mathrm{u}}$, which implies that there exists a u-chaotic continuum $Z$ of $f$, contained in $\mathrm{Cl}\left(V^{\mathrm{u}}(x)\right)$. 
(3.10) Lemma ([16, the proof of (3.2)]). Let $f: G \rightarrow G$ be a map of a graph $G$ such that $f$ has sensitive dependence on initial conditions and let $X=(G, f)$. Then there is $\tau>0$ such that if $A \in C(X)$ and $\operatorname{diam} A \leq \tau$, then $A \in \mathbb{V}^{\mathrm{u}}$.

(3.11) Lemma $([17,(1.6)])$. Let $f$ be a continuum-wise expansive homeomorphism of a Peano continuum $X$. Then there is $\delta>0$ such that for each $x \in X$, there exist $A, B \in C(X)$ such that $x \in A \cap B, \operatorname{diam} A=\delta=\operatorname{diam} B$, $A \in \mathbb{V}^{\mathrm{s}}$ and $B \in \mathbb{V}^{\mathrm{u}}$.

By (3.10), (3.11) and Claim (*) in the proof of (3.6), we obtain the following.

(3.12) Corollary. Let $f: G \rightarrow G$ be a map of a graph $G$ such that $f$ has sensitive dependence on initial conditions. Then for each $\widetilde{x} \in(G, f)$, $\mathrm{Cl}\left(V^{\mathrm{u}}(\widetilde{x})\right)$ contains a u-chaotic continuum of $\widetilde{f}$, and there is no s-chaotic continuum of $\tilde{f}$.

(3.13) Corollary. Let $f$ be a continuum-wise expansive homeomorphism of a Peano continuum $X$. Then for both $\sigma=\mathrm{s}$ and $\mathrm{u}$ and for each $x \in X, \mathrm{Cl}\left(V^{\sigma}(x)\right)$ contains a $\sigma$-chaotic continuum of $f$.

(3.14) Remark. In $[18,(2.3)]$, we showed that there is an expansive homeomorphism $f$ of a one-dimensional continuum $X$ such that there is an open dense set $U$ of $X$ so that if $x \in U$, then $W^{\sigma}(x)=V^{\sigma}(x)=\{x\}(\sigma=\mathrm{s}$ and $\mathrm{u}$ ). Hence $\mathrm{Cl}\left(V^{\sigma}(x)\right)$ does not contain a chaotic continuum of $f$.

A homeomorphism $f$ of a continuum $X$ is continuum-wise fully expansive (resp. positively continuum-wise fully expansive) [17] if for any $\varepsilon_{1}, \varepsilon_{2}>0$, there is $N=N\left(\varepsilon_{1}, \varepsilon_{2}\right)>0$ such that if $A \in C(X)$ and $\operatorname{diam} A \geq \varepsilon_{2}$, then either $d_{\mathrm{H}}\left(f^{n}(A), X\right)<\varepsilon_{1}$ for each $n \geq N$ or $d_{\mathrm{H}}\left(f^{-n}(A), X\right)<\varepsilon_{1}$ for each $n \geq N$ (resp. $d_{\mathrm{H}}\left(f^{n}(A), X\right)<\varepsilon_{1}$ for each $\left.n \geq N\right)$.

(3.15) Proposition. If $f$ is a continuum-wise fully expansive homeomorphism of a continuum $X$, then $X$ is a chaotic continuum of $f$.

Proof. By [17, (3.2)], either $\sigma=\mathrm{s}$ or $\sigma=\mathrm{u}$ has the property that for each $x \in X$ there is $A(x) \in \mathbb{V}^{\sigma}(\delta ; \varepsilon)$ with $x \in A(x)$, where $\varepsilon, \delta$ are as in (3.2). Suppose that $\sigma=\mathrm{s}$. For each $n \geq 0$, choose $A\left(f^{n}(x)\right) \in \mathbb{V}^{\mathrm{s}}(\delta ; \varepsilon)$ as above. Then $f^{-n}\left(A\left(f^{n}(x)\right)\right) \subset V^{\mathrm{s}}(x)$. Since $f$ is continuum-wise fully expansive, we know that $\lim _{n \rightarrow \infty} f^{-n}\left(A\left(f^{n}(x)\right)\right)=X$. Hence $\operatorname{Cl}\left(V^{\mathrm{s}}(x)\right)=X$ for each $x \in X$. This implies that $X$ is an s-chaotic continuum of $f$.

(3.16) EXAMPLE. In (3.6), we cannot replace the assumption that $f$ is a continuum-wise expansive homeomorphism with the assumption that $f$ is a homeomorphism which has sensitive dependence on initial conditions. 
(1) Let $S^{1}$ be the unit circle and $I$ the unit interval. Let $r_{\alpha}$ denote the rotation through $2 \pi \alpha$ on $S^{1}$. Define a homeomorphism $f: S^{1} \times I \rightarrow S^{1} \times I$ by $f(x, t)=\left(r_{t \alpha}(x), t\right)$ for $x \in S^{1}$ and $t \in I$. Then $f$ has sensitive dependence on initial conditions. Note that $W^{\sigma}(x)=V^{\sigma}(x)=\{x\}$. Hence there is no weakly chaotic continuum of $f$.

(2) Let $D=\{0,1\}$ and $C=\prod_{-\infty<n<\infty} D_{n}$, where $D_{n}=D$. Let $\sigma$ : $C \rightarrow C$ be the shift of $C$, i.e., $\sigma\left(\left(a_{n}\right)_{n}\right)=\left(a_{n-1}\right)_{n}$. Consider the cone $X$ of $C$, i.e., $X=(C \times I) /(C \times\{0\})$. $X$ is called a Cantor fan. Define a homeomorphism $f: X \rightarrow X$ by $f([x, t])=[\sigma(x), \sqrt{t}]$ for $x \in C$ and $t \in I$. Then $f$ has sensitive dependence on initial conditions, but there is no weakly chaotic continuum of $f$. Note that $X$ is one-dimensional.

(3.17) Proposition. If $Z$ is a chaotic continuum of a continuum-wise expansive homeomorphism $f$ of a compactum $X$, then both $f$ and $f^{-1}$ have sensitive dependence on initial conditions on $Z$.

4. Continuum-wise expansive homeomorphisms are chaotic in the sense of $\mathrm{Li}$ and Yorke on almost all Cantor sets of chaotic continua. In this section, we prove the following theorem.

(4.1) THEOREM. Let $f$ be a homeomorphism of a compactum $X$ with $\operatorname{dim} X>0$. If there exists a $\sigma$-chaotic continuum $Z$ of $f$, then $f$ or $f^{-1}$ is chaotic in the sense of Li and Yorke on almost all Cantor sets $C \subset Z$ according as $\sigma=\mathrm{s}$ or $\mathrm{u}$.

To prove (4.1), we need the following.

A subset $E$ of a space $X$ is called an $F_{\sigma^{-}}$set in $X$ if $E$ is a countable union of closed subsets of $X$, and $E$ is of the first category if $E=\bigcup_{n=1}^{\infty} E_{n}$ where $E_{n}$ is nowhere dense, i.e., $\operatorname{Int}_{X} \operatorname{Cl}\left(E_{n}\right)=\emptyset$ (see [20]).

We will use a theorem of K. Kuratowski on independent sets [21]. A subset $F$ of a space $X$ is said to be independent in $R \subset X^{n}$ if for every system $x_{1}, \ldots, x_{n}$ of different points of $F$ the point $\left(x_{1}, \ldots, x_{n}\right) \in F^{n}$ never belongs to $R$. In [21], Kuratowski proved the following useful theorem.

(4.2) Theorem ([21, Main theorem and Corollary 3]). If $X$ is a complete space and $R \subset X^{n}$ is an $F_{\sigma}$-set of the first category, then the set $J(R)$ of all compact subsets $F$ of $X$ independent in $R$ is a dense $G_{\delta}$-set in the space $2^{X}$ of all compact subsets of $X$. Moreover, if $X$ has no isolated points, then almost all Cantor sets in $X$ are independent in $R$.

Pro of of (4.1). Suppose that $\sigma=$ s. Since $Z$ is an s-chaotic continuum of $f$, there is $\tau>0$ such that

(*) for any $x \in Z$ and any neighborhood $U$ of $x$ in $X$ there is $y \in U \cap Z$ such that $\liminf _{n \rightarrow \infty} d\left(f^{n}(x), f^{n}(y)\right) \geq \tau$. 
Choose $\varrho>0$ such that $0<\varrho<\tau / 5$. Set

$$
R_{1}^{\mathrm{s}}(\tau / 2)=\left\{(x, y) \in Z \times Z \mid \limsup _{n \rightarrow \infty} d\left(f^{n}(x), f^{n}(y)\right) \leq \tau / 2\right\} .
$$

Then $R_{1}^{\mathrm{s}}(\tau / 2)$ is an $F_{\sigma}$-set in $Z \times Z$, because $R_{1}^{\mathrm{s}}(\tau / 2)=\bigcup_{n=0}^{\infty} T_{n}$, where

$$
T_{n}=\left\{(x, y) \in Z \times Z \mid d\left(f^{i}(x), f^{i}(x)\right) \leq \tau / 2 \text { for } i \geq n\right\},
$$

and $T_{n}$ is closed for each $n$. Since $\operatorname{Cl}\left(V^{\mathrm{s}}(z ; Z)\right)=Z$ for each $z \in Z$, by $(*)$, $R_{1}^{\mathrm{s}}(\tau / 2)$ is an $F_{\sigma}$-set of the first category in $Z \times Z$. Next, put

$$
R_{2}^{\mathrm{s}}=\left\{(x, y) \in Z \times Z \mid \liminf _{n \rightarrow \infty} d\left(f^{n}(x), f^{n}(y)\right)>0\right\} .
$$

Let $\mathbb{Q}_{+}$be the set of all positive rational numbers. For each natural number $n \geq 1$ and $\eta \in \mathbb{Q}_{+}$, set

$$
W_{n, \eta}=\left\{(x, y) \in Z \times Z \mid d\left(f^{i}(x), f^{i}(y)\right) \geq \eta \text { for each } i \geq n\right\} .
$$

Then we can easily see that

$$
R_{2}^{\mathrm{s}}=\bigcup_{\eta \in \mathbb{Q}_{+}} \bigcup_{n=1}^{\infty} W_{n, \eta} .
$$

Note that $W_{n, \eta}$ is closed in $Z \times Z$. Since $Z$ is an s-chaotic continuum of $f$, $W_{n, \eta}$ is nowhere dense in $Z \times Z$. Hence $R_{2}^{\mathrm{s}}$ is an $F_{\sigma}$-set of the first category in $Z \times Z$.

Let $\operatorname{Per}(f)$ be the set of periodic points of $f$ and let $\operatorname{Per}_{m}(f)$ be the set of periodic points of $f$ with period $\leq m$. Put

$P^{\mathrm{s}}(\tau / 5)=\{x \in Z \mid$ there is $p \in \operatorname{Per}(f)$ such that

$$
\left.\limsup _{n \rightarrow \infty} d\left(f^{n}(x), f^{n}(p)\right) \leq \tau / 5\right\}
$$

Then $P^{\mathrm{s}}(\tau / 5)=\bigcup_{m=1}^{\infty} P_{m}$, where

$$
P_{m}=\left\{x \in Z \mid \text { there is } p \in \operatorname{Per}_{m}(f)\right. \text { such that }
$$

$$
\left.\limsup _{n \rightarrow \infty} d\left(f^{n}(x), f^{n}(p)\right) \leq \tau / 5\right\} .
$$

Each $P_{m}$ is an $F_{\sigma}$-set in $Z$. In fact, $P_{m}=\bigcup_{n=1}^{\infty} P_{m, n}$, where

$$
\begin{aligned}
& P_{m, n}=\left\{x \in Z \mid \text { there is } p \in \operatorname{Per}_{m}(f)\right. \text { such that } \\
& \left.\qquad d\left(f^{i}(x), f^{i}(p)\right) \leq \tau / 5 \text { for each } i \geq n\right\},
\end{aligned}
$$

and $P_{m, n}$ is closed in $Z$.

Now, we show that each $P_{m, n}$ is nowhere dense. Choose $\eta>0$ such that if $A \subset X$ with $\operatorname{diam} A \leq \eta$, then $\max \left\{\operatorname{diam} f^{i}(A) \mid 0 \leq i \leq m\right\} \leq \tau / 5$. Choose a finite closed cover $\left\{A_{1}, \ldots, A_{r}\right\}$ of $\operatorname{Per}_{m}(f)$ such that diam $A_{j} \leq \eta$ for each $j=1, \ldots, r$. Let $W \neq \emptyset$ be open in $Z$. Since $Z$ is chaotic, there are $x_{1}, x_{2} \in Z \cap W$ such that $\liminf _{i \rightarrow \infty} d\left(f^{i}\left(x_{1}\right), f^{i}\left(x_{2}\right)\right) \geq \tau$. Since for each 
$i \geq 0, \operatorname{diam} f^{i}\left(A_{j}\right) \leq \tau / 5(j=1, \ldots, r)$, for each $j$ there is $x_{k}(k=1$ or 2$)$ such that $\limsup _{i \rightarrow \infty} d\left(f^{i}\left(x_{k}\right), f^{i}(p)\right) \geq 2 \tau / 5$ for all $p \in A_{j}$. Hence

$$
\begin{aligned}
& P_{m, n}\left(A_{j}\right)=\left\{x \in Z \mid \text { there is } p \in A_{j}\right. \text { such that } \\
& \left.\qquad d\left(f^{i}(x), f^{i}(p)\right) \leq \tau / 5 \text { for each } i \geq n\right\}
\end{aligned}
$$

is nowhere dense in $Z$. Since $P_{m, n}=\bigcup_{j=1}^{r} P_{m, n}\left(A_{j}\right)$ is nowhere dense in $Z$, this implies that $P^{\mathrm{s}}(\tau / 5)$ is an $F_{\sigma}$-set of the first category in $Z$.

Put

$$
R=R_{1}^{\mathrm{s}}(\tau / 2) \cup R_{2}^{\mathrm{s}} \cup\left(P^{\mathrm{s}}(\tau / 5) \times Z\right) \cup\left(Z \times P^{\mathrm{s}}(\tau / 5)\right) .
$$

Then $R$ is an $F_{\sigma}$-set of the first category in $Z \times Z$. By Kuratowski's theorem (4.2) on independent sets, for almost all Cantor sets $C \subset Z$, the following condition is satisfied: if $x, y \in C$ and $x \neq y$, then

(i) $\lim \sup _{n \rightarrow \infty} d\left(f^{n}(x), f^{n}(y)\right) \geq \tau / 2>\varrho$,

(ii) $\liminf _{n \rightarrow \infty} d\left(f^{n}(x), f^{n}(y)\right)=0$, and

(iii) $\lim \sup _{n \rightarrow \infty} d\left(f^{n}(x), f^{n}(p)\right) \geq \tau / 5>\varrho$ for all $p \in \operatorname{Per}(f)$.

These conditions are stronger than those for chaos in the sense of $\mathrm{Li}$ and Yorke. Therefore $f$ is chaotic on $C$ in the sense of $\mathrm{Li}$ and Yorke. The case of $\sigma=\mathrm{u}$ is similar. This completes the proof.

By (3.6) and (4.1), we obtain the following

(4.3) Corollary. If $f$ is a continuum-wise expansive homeomorphism of a compactum $X$ with $\operatorname{dim} X>0$, then there exists a subcontinuum $Z$ of $X$ such that either $f$ or $f^{-1}$ is chaotic in the sense of Li and Yorke on almost all Cantor sets $C \subset Z$.

In [18], we proved that if $f$ is an expansive homeomorphism of a compactum $X$ with $\operatorname{dim} X>0$, then the decomposition $\left\{W^{\sigma}(x) \mid x \in X\right\}(\sigma=\mathrm{s}$ and $\mathrm{u}$ ) is uncountable, and moreover there is $\sigma(=\mathrm{s}$ or $\mathrm{u})$ and $\varrho>0$ such that there exists a perfect closed set $Z \subset X$ such that for almost all Cantor sets $C \subset Z$, the following conditions are satisfied: (I) if $x \in Z$, then $W^{\sigma}(x)$ contains some $A_{x} \in \mathbb{V}^{\sigma}$ with $x \in A_{x}$ and $\operatorname{diam} A_{x} \geq \varrho$, and (II) if $x, y \in C$ and $x \neq y$, then $W^{\sigma}(x) \neq W^{\sigma}(y)$. We say that such a closed set $Z$ has $\sigma$-striped structure (see [18]).

Hence we have the following.

(4.4) Corollary (cf. $[18,(3.1)]$ ). Let $f$ be a homeomorphism of a compactum $X$ with $\operatorname{dim} X>0$. If there exists a $\sigma$-chaotic continuum of $f$, then it has $\sigma$-striped structure.

(4.5) Corollary (cf. [18, (3.1)]). If $f$ is a continuum-wise expansive homeomorphism of a compactum $X$ with $\operatorname{dim} X>0$, then for either $\sigma=\mathrm{s}$ or $\mathrm{u}$, there is a subcontinuum of $X$ which has $\sigma$-striped structure. 
In the case of a general homeomorphism $f: X \rightarrow X$, we do not know whether $W^{\sigma}(x)$ is an $F_{\sigma}$-set in $X$ or not, nor whether

$$
\begin{aligned}
& R_{1}^{\mathrm{s}}=\left\{(x, y) \in X \times X \mid \lim _{n \rightarrow \infty} d\left(f^{n}(x), f^{n}(y)\right)=0\right\} \text { and } \\
& R_{1}^{\mathrm{u}}=\left\{(x, y) \in X \times X \mid \lim _{n \rightarrow \infty} d\left(f^{-n}(x), f^{-n}(y)\right)=0\right\}
\end{aligned}
$$

are $F_{\sigma}$-sets in $X \times X$ or not. Note that if $f$ is expansive, then $W^{\sigma}(x)$ and $R_{1}^{\sigma}$ are $F_{\sigma}$-sets.

Hence, we have the following questions.

(4.6) Question. Let $f$ be a homeomorphism of a compactum $X$ with $\operatorname{dim} X>0$, and suppose that there exists a weakly chaotic continuum $Z$ of $f$. Is then either $f$ or $f^{-1}$ chaotic in the sense of $\mathrm{Li}$ and Yorke on almost all Cantor sets $C \subset Z$ ?

(4.7) Question. Let $f$ be a homeomorphism of a compactum $X$ with $\operatorname{dim} X>0$, and $Z$ a weakly $\sigma$-chaotic continuum of $f$. Does $Z$ have $\sigma$-striped structure?

(4.8) R e mark. In the statements of (4.3) and (4.5), we cannot replace the assumption that $f$ is a continuum-wise expansive homeomorphism with the assumption that $f$ is a homeomorphism with sensitive dependence on initial conditions. Let $f: S^{1} \times I \rightarrow S^{1} \times I$ be as in (1) of (3.16). Then $f$ has sensitive dependence on initial conditions. Note that no nondegenerate subset $D$ of $S^{1} \times I$ is a scrambled set of $f$ or $f^{-1}$. Hence neither $f$ nor $f^{-1}$ is chaotic in the sense of $\mathrm{Li}$ and Yorke. Also, there is no closed subset $Z$ of $S^{1} \times I$ which has $\sigma$-striped structure.

(4.9) Corollary. Let $f$ be a positively continuum-wise expansive map of a compactum $X$ with $\operatorname{dim} X>0$. Then there is an uncountable set $\mathcal{S}$ of sequences $\left\{x_{n}\right\}_{n=0}^{\infty}$ in $X$ such that for some $\eta>0$, if $\left\{x_{n}\right\},\left\{y_{n}\right\} \in \mathcal{S}$ and $\left\{x_{n}\right\} \neq\left\{y_{n}\right\}$, then

(1) $f\left(x_{n+1}\right)=x_{n}(n \geq 0)$

(2) $\limsup _{n \rightarrow \infty} d\left(x_{n}, y_{n}\right)>\eta$,

(3) $\liminf _{n \rightarrow \infty} d\left(x_{n}, y_{n}\right)=0$,

(4) $\limsup _{n \rightarrow \infty} d\left(x_{n}, p\right)>\eta$ for any periodic point $p$ of $f$.

Proof. By $[16,(3.1)]$, the shift map $\tilde{f}:(X, f) \rightarrow(X, f)$ is a positively continuum-wise expansive homeomorphism. By [16, (5.7)], $\operatorname{dim}(X, f)>0$. Then $\mathbb{V}^{\mathrm{u}}$ contains a nondegenerate element. By (3.8), there exists a u-chaotic continuum of $\widetilde{f}$. Then the conclusion follows from the conditions (i)-(iii) as in the proof of (4.1).

(4.10) Corollary. Let $f: G \rightarrow G$ be a map of a graph $G$ which has sensitive dependence on initial conditions. Then there is an uncountable set $\mathcal{S}$ of sequences $\left\{x_{n}\right\}_{n=0}^{\infty}$ in $G$ satisfying (1)-(4) of (4.9). 
Proof. By $[16,(3.8)]$, for graphs, sensitive dependence on initial conditions coincides with positive continuum-wise expansiveness. Hence (4.10) follows from (4.9).

5. Chaotic continua and indecomposability. In this section, we consider the indecomposability of chaotic continua. In [14] and [16], we proved that if a homeomorphism $f$ of a tree-like continuum $X$ is (continuum-wise) expansive, then $X$ contains an indecomposable continuum. In [15] and [16], we proved that if a homeomorphism $f$ of a continuum $X$ is (continuum-wise) expansive and there is a finite family $\mathbb{F}$ of graphs such that $X$ is $\mathbb{F}$-like, then $X$ contains an indecomposable continuum.

Let $X$ be a continuum and let $p \in X$. Then the set $c(p)=\{x \in X \mid$ there is a proper subcontinuum $A$ of $X(A \varsubsetneqq X)$ containing $p$ and $x\}$ is called the composant of $X$ containing $p$. Note that $c(p)$ is a connected subset of $X$ containing $p$, and $c(p)$ is dense in $X$. A continuum $X$ is indecomposable [20] if there are no two proper subcontinua $A$ and $B$ of $X$ such that $A \cup B$ $=X$.

Let $\mathbb{F}$ be a family of compact polyhedra. A continuum $X$ is $\mathbb{F}$-like if for any $\varepsilon>0$, there is an onto map $f: X \rightarrow P$ for some $P \in \mathbb{F}$ such that $\operatorname{diam} f^{-1}(y)<\varepsilon$ for each $y \in P$. Note that a compactum $X$ is $\mathbb{F}$-like if and only if $X=\operatorname{invlim}\left\{K_{n}, f_{n}\right\}$, where $K_{n} \in \mathbb{F}$ and $f_{n}: K_{n+1} \rightarrow K_{n}$ is onto for each $n=0,1, \ldots$ If $\mathbb{F}=$ all trees $\}$, then an $\mathbb{F}$-like continuum $X$ is called a tree-like continuum. Note that a continuum $X$ is one-dimensional if and only if there is a (countable) family $\mathbb{F}$ of graphs such that $X$ is $\mathbb{F}$-like.

Let $X$ be a compactum and let $A$ be a subcontinuum of $X$. We define an index $T(A ; X)$, which is a natural number or $\infty$, by the following conditions: $T(A ; X)<n$ if and only if there are no $n$ subcontinua $B_{1}, \ldots, B_{n}$ of $X$ such that for each $i, A \cap B_{i} \neq \emptyset, B_{i}-A \neq \emptyset$ and $B_{i} \cap B_{j}=\emptyset(i \neq j)$. If $T(A ; X) \geq n$ for all $n$, then we define $T(A ; X)=\infty$. Put $T(X)=\max \{T(A ; X) \mid A$ is a subcontinuum of $X$, i.e., $A \in C(X)\}$. Note that if $\operatorname{dim} C(X)<\infty$, then $T(X)<\infty$.

Note that if $X$ is an indecomposable continuum, then (1) for each $x \in$ $X$, the composant $c(x)$ is dense in $X$ and (2) for each $x \in X$ and each neighborhood $U$ of $x$ in $X$, there is a point $x^{\prime} \in U$ such that $c(x) \cap c\left(x^{\prime}\right)=\emptyset$ (see [20]). These properties are similar to those in the definition of a weakly chaotic continuum. We are interested in the relations between (continuumwise) expansive homeomorphisms and indecomposability.

One may ask whether chaotic continua of (continuum-wise) expansive homeomorphisms are indecomposable. In general, they are not. In fact, consider an Anosov diffeomorphism $f$ of the 2 -torus $T^{2}$. Then $T^{2}$ is a $\sigma$-chaotic continuum of $f$ for both $\sigma=\mathrm{s}$ and $\mathrm{u}$ (see (2.1)). Note that if a continuum $X$ admits a positively continuum-wise fully expansive homeomorphism, then 
$X$ is itself a u-chaotic continuum of $f$ (see (3.15)), and $X$ is indecomposable (see $[17,(4.5)])$.

Moreover, one may ask the following question: if $f$ is a positively continuum-wise expansive homeomorphism of a compactum $X$ with $\operatorname{dim} X$ $>0$, is it true that each chaotic continuum of $f$ is indecomposable? Also, this question has a negative answer. Let $\widetilde{f}:\left(S^{1}, f\right) \rightarrow\left(S^{1}, f\right)$ be the shift homeomorphism of the 2-adic solenoid $\left(S^{1}, f\right)$ as in $(2.1)$. Then $\tilde{f} \times \widetilde{f}$ : $\left(S^{1}, f\right) \times\left(S^{1}, f\right) \rightarrow\left(S^{1}, f\right) \times\left(S^{1}, f\right)$ is clearly positively continuum-wise expansive, and $X=\left(S^{1}, f\right) \times\left(S^{1}, f\right)$ is a u-chaotic continuum of $\tilde{f} \times \tilde{f}$, but $X$ is not indecomposable. However, the following problem in the one-dimensional case remains open.

(5.1) Problem. Suppose that $f$ is a (positively) continuum-wise expansive homeomorphism of a one-dimensional compactum $X$ and $Z$ is a chaotic continuum of $f$. Is $Z$ indecomposable? (Note that the converse assertion is not true; see (5) of (2.1).)

As a partial answer to this problem, we have the following theorem.

(5.2) Theorem. Let $f$ be a homeomorphism of a compactum $X$ with $\operatorname{dim} X>0$. If $T(X)<\infty$ and there exists a chaotic continuum of $f$, then it is indecomposable.

Proof. Suppose that $T(X)<n<\infty$ and $Z$ is a $\sigma$-chaotic continuum of $f$. Suppose, on the contrary, that $Z$ is decomposable. Then there is a proper subcontinuum $A$ of $Z$ such that $\operatorname{Int}_{Z} A \neq \emptyset$. Since $V^{\sigma}(x ; Z)$ is dense in $Z$ for each $x \in Z$, by (4.4) we can easily choose $n$ subcontinua $B_{1}, \ldots, B_{n}$ of $Z$ such that for each $i, A \cap B_{i} \neq \emptyset, B_{i}-A \neq \emptyset$ and $B_{i} \cap B_{j}=\emptyset(i \neq j)$. Then $T(X) \geq T(A ; X) \geq n$. This is a contradiction.

(5.3) COROLlary. Let $f$ be a homeomorphism of a compactum $X$ with $\operatorname{dim} X>0$ and let $\mathbb{F}$ be a finite family of graphs. If $X$ is $\mathbb{F}$-like and there exists a chaotic continuum of $f$, then it is indecomposable.

Proof. It suffices to show that $T(X)<\infty$. Since $\mathbb{F}$ is a finite family of graphs, we can choose a natural number $n$ such that $\max \{T(G) \mid G \in \mathbb{F}\}$ $<n$. Suppose, on the contrary, that $T(X) \geq n$. Then there is a subcontinuum $A$ of $X$ and $n$ subcontinua $B_{1}, \ldots, B_{n}$ of $X$ such that for each $i, A \cap B_{i} \neq \emptyset$, $B_{i}-A \neq \emptyset$, and $B_{i} \cap B_{j}=\emptyset(i \neq j)$. If we choose a sufficiently small $\varepsilon>0$ and an onto map $g: X \rightarrow G \in \mathbb{F}$ such that $\operatorname{diam} g^{-1}(y)<\varepsilon$ for each $y \in G$, then for each $i, g(A) \cap g\left(B_{i}\right) \neq \emptyset, g\left(B_{i}\right)-g(A) \neq \emptyset$ and $g\left(B_{i}\right) \cap g\left(B_{j}\right)=\emptyset(i \neq j)$. This implies that $T(G) \geq n$, which is a contradiction.

(5.4) Corollary (cf. $[15,(3.1)]$ and $[16,(6.9)])$. Let $f$ be a continuumwise expansive homeomorphism of a compactum $X$ with $\operatorname{dim} X>0$. If there 
is a finite family $\mathbb{F}$ of graphs such that $X$ is $\mathbb{F}$-like, then each chaotic continuum of $f$ is indecomposable. In particular, if $f: G \rightarrow G$ is a map of a graph $G$ such that $f$ has sensitive dependence on initial conditions, then each chaotic continuum of the shift map $\widetilde{f}:(G, f) \rightarrow(G, f)$ is indecomposable.

In [3, Theorem 5], Curry proved that if $X$ is a plane tree-like continuum so that for any $\varepsilon>0, X$ has two disjoint $\varepsilon$-dense subcontinua, then $X$ is either indecomposable or the union of two indecomposable continua.

(5.5) COROLlary. Let $f$ be a continuum-wise expansive homeomorphism of a plane tree-like continuum $X$. Then every chaotic continuum of $f$ is either indecomposable or the union of two indecomposable continua.

The author wishes to thank the referee for his helpful comments.

\section{References}

[1] N. Aoki, Topological dynamics, in: Topics in General Topology, K. Morita and J. Nagata (eds.), Elsevier, 1989, 625-740.

[2] B. F. Bryant, Unstable self-homeomorphisms of a compact space, Thesis, Vanderbilt University, 1954.

[3] S. B. Curry, One-dimensional nonseparating plane continua with disjoint $\varepsilon$-dense subcontinua, Topology Appl. 39 (1991), 145-151.

[4] R. Devaney, An Introduction to Chaotic Dynamical Systems, 2nd ed., AddisonWesley, 1989.

[5] W. Gottschalk, Minimal sets: an introduction to topological dynamics, Bull. Amer. Math. Soc. 64 (1958), 336-351.

[6] W. Gottschalk and G. Hedlund, Topological Dynamics, Amer. Math. Soc. Colloq. Publ. 34, Amer. Math. Soc., 1955.

[7] K. Hiraide, Expansive homeomorphisms on compact surfaces are pseudo-Anosov, Osaka J. Math. 27 (1990), 117-162.

[8] W. Hurewicz and H. Wallman, Dimension Theory, Princeton University Press, Princeton, N.J., 1948.

[9] J. F. Jacobson and W. R. Utz, The nonexistence of expansive homeomorphisms of a closed 2-cell, Pacific J. Math. 10 (1960), 1319-1321.

[10] H. Kato, The nonexistence of expansive homeomorphisms of Peano continua in the plane, Topology Appl. 34 (1990), 161-165.

[11] - On expansiveness of shift homeomorphisms in inverse limits of graphs, Fund. Math. 137 (1991), 201-210.

[12] - , The nonexistence of expansive homeomorphisms of dendroids, ibid. 136 (1990), 37-43.

[13] - Embeddability into the plane and movability on inverse limits of graphs whose shift maps are expansive, Topology Appl. 43 (1992), 141-156.

[14] —, Expansive homeomorphisms in continuum theory, ibid. 45 (1992), 223-243.

[15] - Expansive homeomorphisms and indecomposability, Fund. Math. 139 (1991), $49-57$.

[16] - Continuum-wise expansive homeomorphisms, Canad. J. Math. 45 (1993), 576598. 
[17] H. Kato, Concerning continuum-wise fully expansive homeomorphisms of continua, Topology Appl. 53 (1993), 239-258.

[18] - Striped structures of stable and unstable sets of expansive homeomorphisms and a theorem of K. Kuratowski on independent sets, Fund. Math. 143 (1993), 153-165.

[19] H. Kato and K. Kaw amura, A class of continua which admit no expansive homeomorphisms, Rocky Mountain J. Math. 22 (1992), 645-651.

[20] K. Kuratowski, Topology, Vol. II, Academic Press, New York, 1968.

[21] - Applications of Baire-category method to the problem of independent sets, Fund. Math. 81 (1974), 65-72.

[22] T. Y Li and J. A. Yorke, Period three implies chaos, Amer. Math. Monthly 82 (1975), 985-992.

[23] R. Mañé, Expansive homeomorphisms and topological dimension, Trans. Amer. Math. Soc. 252 (1979), 313-319.

[24] S. B. Nadler, Jr., Hyperspaces of Sets, Pure and Appl. Math. 49, Dekker, New York, 1978.

[25] R. V. Plykin, Sources and sinks of A-diffeomorphisms of surfaces, Math. USSR-Sb. 23 (1974), 233-253.

[26] W. Reddy, The existence of expansive homeomorphisms of manifolds, Duke Math. J. 32 (1965), 627-632.

[27] W. Utz, Unstable homeomorphisms, Proc. Amer. Math. Soc. 1 (1950), 769-774.

[28] P. Walters, An Introduction to Ergodic Theory, Graduate Texts in Math. 79, Springer, 1982.

[29] R. F. Williams, A note on unstable homeomorphisms, Proc. Amer. Math. Soc. 6 (1955), 308-309.

Current address:

FACULTY OF INTEGRATED ARTS AND SCIENCES HIROSHIMA UNIVERSITY

1-7-1 KAGAMIYAMA

HIGASHI-HIROSHIMA, 724 JAPAN
INSTITUTE OF MATHEMATICS UNIVERSITY OF TSUKUBA

IBARAKI 305

JAPAN

Received 17 May 1993;

in revised form 15 January 1994 